\title{
Dynamics of killer $\mathbf{T}$ cell inflation in viral infections
}

\author{
Dominik Wodarz $^{1, *}$, Sophie Sierro ${ }^{2}$ and Paul Klenerman ${ }^{2}$ \\ ${ }^{1}$ Department of Ecology and Evolutionary Biology, University of California, \\ 321 Steinhaus Hall, Irvine, CA 92697, USA \\ ${ }^{2}$ Nuffield Department of Clinical Medicine, Peter Medawar Building for Pathogen Research, \\ University of Oxford, Oxford OX1 $3 S Y, U K$
}

\begin{abstract}
Upon acute viral infection, a typical cytotoxic T lymphocyte (CTL) response is characterized by a phase of expansion and contraction after which it settles at a relatively stable memory level. Recently, experimental data from mice infected with murine cytomegalovirus (MCMV) showed different and unusual dynamics. After acute infection had resolved, some antigen specific CTL started to expand over time despite the fact that no replicative virus was detectable. This phenomenon has been termed as 'CTL memory inflation'. In order to examine the dynamics of this system further, we developed a mathematical model analysing the impact of innate and adaptive immune responses. According to this model, a potentially important contributor to CTL inflation is competition between the specific CTL response and an innate natural killer (NK) cell response. Inflation occurs most readily if the NK cell response is more efficient than the CTL at reducing virus load during acute infection, but thereafter maintains a chronic virus load which is sufficient to induce CTL proliferation. The model further suggests that weaker NK cell mediated protection can correlate with more pronounced CTL inflation dynamics over time. We present experimental data from mice infected with MCMV which are consistent with the theoretical predictions. This model provides valuable information and may help to explain the inflation of CMV specific CD $8+\mathrm{T}$ cells seen in humans as they age.
\end{abstract}

\section{Keywords: mathematical models; virus dynamics; CMV; CTL memory inflation;} competition

\section{INTRODUCTION}

CD8 $+\mathrm{T}$ cell or cytotoxic $\mathrm{T}$ lymphocyte (CTL) responses play an important role in the resolution of viral infections. Activated antigen specific $\mathrm{CD} 8+\mathrm{T}$ cells exert their functions by lysing infected cells and/or by secreting soluble mediators which inhibit viral replication. Following non-persistent viral infections, antigen specific naive CD8 + T cells are primed and expand. The expansion phase is followed by a contraction phase during which $95 \%$ of antigen specific effector CD $8+\mathrm{T}$ cells die. Thereafter, antigen specific CD $8+\mathrm{T}$ cells settle at a relatively stable level and decline only at a very slow rate (Wherry \& Ahmed 2004). This is called CD8+T cell memory and can be maintained in the absence of antigenic stimulation (Kaech et al. 2002). After acute infection, memory specific for a range of epitopes derived from different viral proteins is often observed.

A different pattern of CTL dynamics has been observed after murine cytomegalovirus (MCMV) infection (Holtappels et al. 2002; Karrer et al. 2003). In murine models, $\mathrm{CD} 8+\mathrm{T}$ cells have been shown to be important for both resolution of acute CMV disease and

*Author for correspondence (dwodarz@uci.edu). maintenance of virus latency. Similar observations have been made in human infection (Bunde et al. 2005). In MCMV, viral reactivation is initiated from the immediate early (IE) gene complex during latent infection (Kurz et al. 1999). It is thought that CTL specific for the IE gene products control reactivation, because the full replication cycle of the virus is usually not completed (Reddehase 2002). While some CTL responses specific for MCMV showed the typical stable memory pattern described above, CTL directed against other epitopes followed an unusual dynamics: the acute response is characterized by rapid expansion of antigen specific CD $8+\mathrm{T}$ cells, followed by a contraction phase. Thereafter, however, a steady accumulation of the specific CTL during the latent phase of the infection can be observed. Similar late expansion has been observed in responses against subdominant epitopes (endogenous or presened from recombinant viruses) which are barely detectable in the acute phase (Karrer et al. 2004; Munks et al. 2006). Up to $20 \%$ of all CD8 $+\mathrm{T}$ cells can be specific for one epitope 1 year after infection (Karrer et al. 2003), and the expansion comprises changes in absolute numbers, as well as proportions of CD8 T cells, in all organs studied (especially non-lymphoid organs such as lung, liver and salivary gland; Karrer et al. 2003; 
Sierro et al. 2005). These particular dynamics have been termed as 'memory inflation', and could explain why very large numbers of CTL specific for human CMV are observed in seropositive individuals long after the resolution of primary infection (Khan et al. 2002, 2004; Komatsu et al. 2003). It has been suggested that these very large expansions may compromise immune responsiveness against other infections, such as EBV or vaccines, and may therefore contribute to the increased mortality rate associated with CMV seropositivity in the elder people (Khan et al. 2004).

While the observation of memory inflation is well characterized experimentally, the mechanisms which contribute to these dynamics have yet to be explored. Responses to many epitopes do not appear to undergo inflationary dynamics. Detailed analysis of the memory pool suggests that these do not appear to re-encounter antigen, as judged by markers of activation and maturation. Experimentally, it is difficult to examine antigen representation during latency in vivo, but most observers conclude that virally encoded immunoevasins, which downregulate antigen processing as viral replication proceeds in an infected cell probably play a major role in preventing recognition of such epitopes. The power of the immunoevasin pathways is such that relatively immunodominant responses generated acutely may fail to protect mice against viral re-challenge in a bone marrow transplant model.

For the other epitopes, however, the unusual dynamics of the CMV response have yet to be adequately explained. Since the host-virus interactions comprise very low levels of replication in diverse tissues they are not readily amenable to experimental measurement. Thus, we have formulated a simple mathematical model to examine this process. Using this, we propose a mechanism which could explain some key aspects of memory inflation. We find that memory inflation can be promoted by the competition dynamics between CMVspecific CD8+ T cells and innate natural killer (NK) cell responses. $\mathrm{CD} 8+\mathrm{T}$ cell inflation for specific epitopes occurs if the NK cells reduce virus load more quickly than the antigen specific CD $8+\mathrm{T}$ cells during acute infection, but maintain a chronic virus load which allows for subsequent CD8 $+\mathrm{T}$ cell expansion to higher levels. In particular, the extent of memory inflation can depend on the efficacy with which NK cells control the infection. Weaker NK cell responses can correlate with more pronounced inflation dynamics. The theoretical results are supported by further experimental data.

\section{MATERIAL AND METHODS}

\subsection{Basic model of CMV infection}

The model which describes the basic dynamics of CMV infection is given by the following set of ordinary differential equations:

$$
\begin{aligned}
\frac{\mathrm{d} x}{\mathrm{~d} t} & =\lambda-\mathrm{d} x-\beta x v-\gamma x v, \\
\frac{\mathrm{d} y_{0}}{\mathrm{~d} t} & =\beta x v-a_{0} y_{0}-\eta y_{0}+\phi L,
\end{aligned}
$$

$$
\begin{aligned}
\frac{\mathrm{d} y_{1}}{\mathrm{~d} t} & =\eta y_{0}-a_{1} y_{1}, \\
\frac{\mathrm{d} L}{\mathrm{~d} t} & =\gamma x v-\phi L-\mathrm{d} L, \\
\frac{\mathrm{d} v}{\mathrm{~d} t} & =k y_{1}-u v .
\end{aligned}
$$

The meaning of the variables is as follows: susceptible host cells, $x$; infected cells which express early-viral gene products, $y_{0}$; infected cells which express late-viral gene products, $y_{1}$; latently infected cells, $L$; and free virus particles, $v$.

Two outcomes are possible in this model. If the infection fails to get established, the population of susceptible host cells remains at the equilibrium level $x=\lambda / d$, while all other populations are extinct. Alternatively, the infection can become established and the equilibrium expressions for this outcome are given by complicated expressions which are not written out here. The infection becomes established if the basic reproductive ratio of the virus, $R_{0}$, is greater than one. The basic reproductive ratio is given by

$$
R_{0}=\frac{\lambda \eta}{d_{1} a_{1}\left(a_{0}+\eta\right)}\left(\beta+\frac{\gamma \phi}{\phi+d}\right) .
$$

\subsection{Including a CTL response}

The dynamics of the CTL response is modelled in two parts. First, upon encounter with antigen, it is assumed that the naive CTL undergo programmed expansion which involves eight cell divisions, and the generation of effector and effector memory cells. Subsequently, it is assumed that repeated encounter with antigen results in further CTL expansion and the generation of effector cells. This process which occurs in chronic infection is approximated with a simple predator-prey type equation.

Programmed CTL expansion during acute infection is modelled by the following set of differential equations which have been proposed by Wodarz \& Thomsen (2005):

$$
\left.\begin{array}{c}
\frac{\mathrm{d} m_{n}}{\mathrm{~d} t}=2 r m_{n-1}-r m_{n} \\
\vdots \\
\frac{\mathrm{d} m_{1}}{\mathrm{~d} t}=2 r m_{0}-r m_{1} \\
\frac{\mathrm{d} m_{0}}{\mathrm{~d} t}=-r m_{0}
\end{array}\right\} .
$$

The variable $m$ denotes the number of acute CTL which have not yet developed effector activity. We start with the population $m_{0}$. These are naive CTL which have just been activated by CMV. These cells start to divide. The CTL which have undergone $i$ cell divisions are denoted by $m_{i}$. In accordance with experimental data we assume that CTL undergo eight divisions before they attain effector activity. That is, $n=8$.

After the eight cell divisions, the CTL are assumed to attain effector function which can subsequently become effector memory cells and repeatedly give rise 
to further effectors upon continuous antigenic stimulation. This is modelled by the following set of equations (Nowak \& Bangham 1996):

$$
\begin{aligned}
\frac{\mathrm{d} x}{\mathrm{~d} t} & =\lambda-d x-\beta x v-\gamma x v, \\
\frac{\mathrm{d} y_{0}}{\mathrm{~d} t} & =\beta x v-a_{0} y_{0}-\eta y_{0}+\phi L-p_{\mathrm{a}} y_{0} z_{\mathrm{a}}, \\
\frac{\mathrm{d} y_{1}}{\mathrm{~d} t} & =\eta y_{0}-a_{1} y_{1}-p_{\mathrm{a}} y_{1} z_{1}, \\
\frac{\mathrm{d} L}{\mathrm{~d} t} & =\gamma x v-\phi L-\mathrm{d} L \\
\frac{\mathrm{d} v}{\mathrm{~d} t} & =k y_{1}-u v, \\
\frac{\mathrm{d} z_{\mathrm{a}}}{\mathrm{d} t} & =\alpha m_{n}+c_{\mathrm{a}}\left(y_{0}+y_{1}\right) z_{\mathrm{a}}-b_{\mathrm{a}} z_{\mathrm{a}} .
\end{aligned}
$$

The CMV specific effector CTL are denoted by $z_{\mathrm{a}}$ (subscript a stands for adaptive to set it apart from the innate NK cell response introduced in \$2.3). They are produced with a rate $\alpha m_{n}$ by differentiation from precursor cells which have undergone programmed expansion. Upon stimulation with antigen derived from early- and late-stage infected cells, the CTL proliferate further with a rate $c_{\mathrm{a}}$. They die with a rate $b_{\mathrm{a}}$. The effector CTL are assumed to lyse both early- and latestage infected cells with a rate $p_{\text {a }}$. Non-lytic activity could be easily incorporated into the model and would not change the outcomes considered here.

If $c_{\mathrm{a}}\left(y_{0}^{*}+y_{1}^{*}\right)>b$, the system converges towards an equilibrium which describes persistent infection controlled by CTL $\left(y_{0}^{*}+y_{1}^{*}\right.$ denote the abundance of infected cells in the absence of immunity). The mathematical expressions for the equilibrium are complicated and are not written out here.

\subsection{Modelling the NK cell response}

In the following model, an NK cell response is added to the above equations.

$$
\begin{aligned}
\frac{\mathrm{d} x}{\mathrm{~d} t} & =\lambda-\mathrm{d} x-\beta x v-\gamma x v \\
\frac{\mathrm{d} y_{0}}{\mathrm{~d} t} & =\beta x v-a_{0} y_{0}-\eta y_{0}+\phi L-p_{\mathrm{a}} y_{0} z_{\mathrm{a}}-p_{\mathrm{i}} y_{0} z_{\mathrm{i}} \\
\frac{\mathrm{d} y_{1}}{\mathrm{~d} t} & =\eta y_{0}-a_{1} y_{1}-p_{\mathrm{a}} y_{1} z_{\mathrm{a}}-p_{\mathrm{i}} y_{1} z_{\mathrm{i}} \\
\frac{\mathrm{d} L}{\mathrm{~d} t} & =\gamma x v-\phi L-\mathrm{d} L \\
\frac{\mathrm{d} v}{\mathrm{~d} t} & =k y_{1}-u v, \\
\frac{\mathrm{d} z_{\mathrm{a}}}{\mathrm{d} t} & =\alpha m_{n}+c_{\mathrm{a}}\left(y_{0}+y_{1}\right) z_{\mathrm{a}}-b_{\mathrm{a}} z_{\mathrm{a}} \\
\frac{\mathrm{d} z_{\mathrm{i}}}{\mathrm{d} t} & =\xi+c_{\mathrm{i}}\left(y_{0}+y_{1}\right) z_{\mathrm{i}}-b_{\mathrm{i}} z_{\mathrm{i}} .
\end{aligned}
$$

The population of NK cells is denoted by $z_{\mathrm{i}}$ (i stands for innate). It is assumed that reactive NK cells are present independent from the virus. They are produced with a constant rate $\xi$ and dies with a rate $b_{\mathrm{i}}$. Thus, in the absence of infection, NK cells which can react against CMV are present at a level of $\xi / b_{\mathrm{i}}$. In addition, it is assumed that the population of NK cells can undergo clonal expansion upon antigenic stimulation with a rate $c_{\mathrm{i}}$. Since the NK cells, which can attack the virus, are always around even in the absence of an infection there is no condition for the establishment of the NK cell response. In contrast to the CTL, limited antigenic stimulation cannot drive the number of NK cells below a limit.

2.3.1. Mice. Mice were bred and maintained in a conventional animal facility unit at the Department of Zoology, University of Oxford, UK. All experiments were performed using age-matched control female C57BL/6 or $\mathrm{BALB} / \mathrm{B}$ mice and with the permission of the Home Office according to the Animals Scientific Procedures Act of 1986. Mice were infected intravenously (i.v.) with $10^{6}$ pfu MCMV-GP (Karrer et al. 2004).

2.3.2. In vivo depletion of $N K$ cells. The hybridoma secreting anti-NK1.1- (PK136) was used (Seaman et al. 1987). The hybridoma was grown in culture and mAbs were purified by precipitation in saturated ammonium sulphate. The optimal amount of Abs for in vivo depletion was determined in preliminary assays by flow cytometry (data not shown). Mice were injected twice with $300 \mu \mathrm{g}$ of purified PK136 mAb with a one-day interval. Antibodies, injected i.p. in volumes of 100-200 $\mu$ l, were administered 3 and 1 day prior to infection.

2.3.3. Ex vivo detection of antigen-specific T cells using MHC class I tetrameric complexes and flow cytometry. The MHC class I-peptide tetrameric complexes (tetramers) used in the study were produced as described previously (Altman et al. 1996). The following peptide for the $\mathrm{H}-2^{\mathrm{b}}$ haplotype: the LCMV glycoprotein derived epitope gp34 (GP, H2-K ${ }^{\mathrm{b}},{ }^{34}$ AVYNFATC ${ }^{41}$, Hudrisier et al. 1997) was used. For FACS staining, the following antibodies were used: allophycocyanin (APC)- or Peridinin Chlorophyll protein (PerCP)-labelled antimouse CD8 (BD Biosciences).

\section{RESULTS AND DISCUSSION}

\subsection{Modelling basic dynamics of CMV infection}

The model for CMV infection is based on simple virus dynamics equations (Nowak \& Bangham 1996; De Boer \& Perelson 1998; Perelson 2002) and contains the following additional features: (i) the distinction between infected cells which express only viral gene products expressed early in the viral life cycle (such as Immediate early genes) but no infectious virus, and infected cells which express also the viral gene products generated only later in the viral life cycle, along with new viral progeny; (ii) a compartment of latently infected cells. Thus, the model contains the following five variables: susceptible host cells, $x$; infected cells which express early-viral gene products, $y_{0}$; infected cells which express late-viral gene products, $y_{1}$; latently infected cells, $L$; and free virus particles, $v$. It is given by a set of differential 

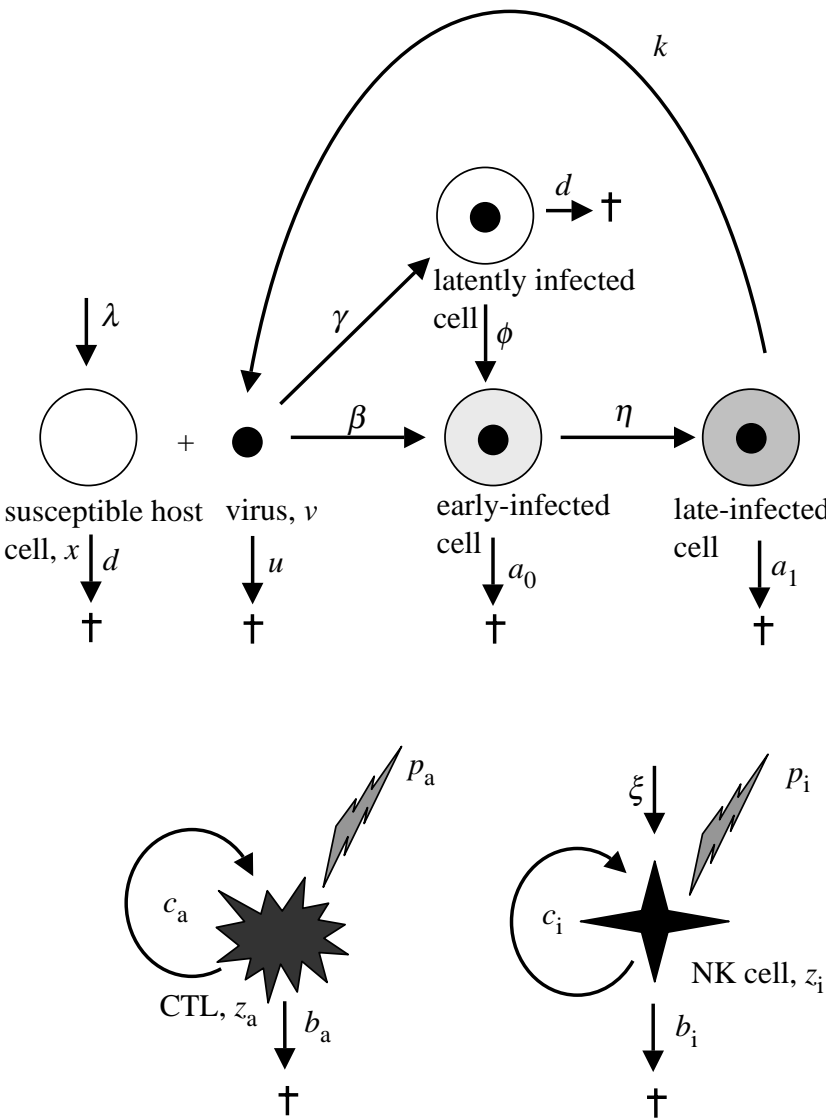

Figure 1. Schematic depicting the basic assumptions which underlie the mathematical model. The basic model consists of five variables. Susceptible host cells, early-infected cells, lateinfected cells, latently infected cells and free virus. The details of the model are explained in the text. When a virus particle meets a susceptible host cell and infection occurs, either a productively infected cell or a latently infected cell is generated. The productively infected cells are subdivided into two populations: those which express early-gene products (early-infected cells) and those which express late-gene products (late-infected cells). Late-infected cells produce new free virus particles and the replication cycle is completed. Latently infected cells are silent but become activated with a certain probability. This gives rise to productively infected cells. In addition to these basic dynamics, the model also contains two types of immune responses. A specific CTL response expands upon antigenic stimulation and exerts antiviral effector activity (through lysis). The second immune response in the model are NK cells. A certain number of NK cells with immediate antiviral activity is always around, and they can also expand in response to antigenic stimulation. The parameters of the models and their meaning are summarized in table 1.

equations which describes the development of these populations over time. The equations are shown schematically in figure $1 a$ and written out in $\S 2$. The meaning of model parameters is summarized in table 1 . Susceptible host cells are produced with a rate $\lambda$ and dies with a rate $d$. Virus infection results in the generation of a productively infected cell with a rate $\beta$, and in the generation of a latently infected cell with a rate $\gamma$. Productively infected cells first express earlyviral gene products (cells denoted by $y_{0}$ ). They die at a rate $a_{0}$, and start expressing late-gene products with a rate $\eta$ (cells denoted by $y_{1}$ ). Infected cells which
Table 1. List of parameters and their meanings. Parameter values chosen for simulations are shown in the figure legends. Parameter values have been chosen for illustrative purposes only. No measurements of the parameters are available so far. Hence, they have not been listed in this table.

\begin{tabular}{ll}
\hline parameter & meaning \\
\hline$\lambda$ & production rate of susceptible host cells \\
$d$ & natural death rate of susceptible host cells \\
$\beta$ & rate of productive infection \\
$\gamma$ & generation rate of latently infected cells \\
$a_{0}$ & death rate of early-infected cells \\
$a_{1}$ & death rate of late-infected cells \\
$\eta$ & rate at which an early-infected cell becomes \\
& a late-infected cell \\
$\phi$ & activation rate of latently infected cells \\
$k$ & rate of virus production by infected cells \\
$u$ & death rate of free virus particles \\
$p_{\mathrm{a}}$ & rate of CTL-mediated killing of infected \\
$p_{\mathrm{i}}$ & cells (a=adaptive) \\
$r$ & rate of NK cell-mediated killing of infected \\
& cells (i=innate) \\
$\alpha$ & rate of antigen-independent programmed \\
& CTL proliferation \\
$c_{\mathrm{a}}$ & rate of CTL effector generation following \\
$b_{\mathrm{a}}$ & programmed proliferation \\
$\xi$ & rate of antigen-driven CTL proliferation \\
$c_{\mathrm{i}}$ & death rate of effector CTL \\
$b_{\mathrm{i}}$ & production rate of NK cells \\
\hline & rate of antigen-drive expansion of NK cells \\
& death rate of NK cells \\
\hline
\end{tabular}

express late-viral gene products die with a rate $a_{1}$ and produce new virus particles with a rate $k$. New virus particles decay with a rate $u$. Latently infected cells die with a rate $d$ and become activated with a rate $\phi$. Activation is assumed to give rise to an infected cell which expresses early-viral gene products, $y_{1}$.

In this basic model two outcomes are possible. Either the infection fails to become established, or we observe persistent infection. Which outcome is observed depends on the basic reproductive ratio of the virus, denoted by $R_{0}$ (Anderson \& May 1991). This measure describes the average number of newly infected cells which are generated by one infected cell when the host is almost virus free. If $R_{0}<1$, we observe extinction of the virus; if $R_{0}>1$, then we observe persistent infection. In our model, the basic reproductive ratio of the virus is the sum of two components: virus replication which occurs during productive infection and virus replication which is induced by the activation of latently infected cells. Mathematical details of the model are given in materials and methods.

\subsection{Modelling $C T L$ responses against $C M V$}

Here, we add an adaptive immune response, denoted by $z_{\mathrm{a}}$. In particular, we consider a CTL response against CMV. This is modelled according to the simplest assumptions where antigenic stimulation induces CTL proliferation and CTL kill infected cells (Nowak \& Bangham 1996; figure 1). It is assumed that CTL can recognize antigen on both types of productively infected cells, that is, those which express early-viral 
gene products but not yet productive of viral progeny, $y_{0}$; and those which are productive of viral progeny, $y_{1}$. Antigen is assumed not to be recognized on latently infected cells. The CTL response model is given in two parts. In the acute phase, it is assumed that naive CTL become stimulated and undergo programmed expansion which involves eight cell divisions and the differentiation into effector and effector memory cells (Mercado et al. 2000; Kaech \& Ahmed 2001; van Stipdonk et al. 2001; Antia et al. 2003; Wodarz \& Thomsen 2005). Once effectors and memory cells have been generated, the CTL dynamics are modelled by a simple predator-prey type equation: Upon antigenic stimulation (from both types of productively infected cells), CTL proliferate with a rate $c_{\mathrm{a}}$. They die with a rate $b_{\mathrm{a}}$. CTL kill both types of productively infected cells $\left(y_{0}\right.$ and $\left.y_{1}\right)$ with a rate $p_{\mathrm{a}}$. Apart from killing, CTL are known to secrete soluble factors that inhibit viral replication in cells without lysis (Guidotti et al. 1999). According to extensive computer simulations and previous modelling experience (Wodarz \& Nowak 2000; Wodarz et al. 2002), adding this mode of activity does not change the basic results derived here. For simplicity, we only included one mode of effector activity, that is, CTL-mediated lysis.

The reason to use predator-prey equations in the post-acute phase of the infection is that in the context of virus persistence, the CTL are likely to cycle between the effector and memory phenotypes. This should be accurately described by the simple equations. The full mathematical model is given in $§ 2$.

If the degree of antigenic stimulation is sufficiently strong, the specific CTL response becomes established in the long term. This occurs if $c_{\mathrm{a}}\left(y_{0}^{*}+y_{1}^{*}\right)>b_{\mathrm{a}}$, where $y_{0}^{*}$ and $y_{1}^{*}$ denote the equilibrium number of infected cells which express early- and late-viral gene products in the absence of CTL. In this case, the population of CTL grows and starts to downregulate virus load. In a typical simulation, the CTL response peaks and subsequently declines until it reaches a steady state which may correspond to the memory phase (figure $2 a$ ). The number of productively infected cells and the amount of free virus at this steady state is a function of the strength of the immune response. The stronger the CTL response, the lower the number of productively infected cells and the amount of free virus. If the immune response is strong, then most of the virus will persist in the form of latently infected cells. As latent infection is also generated by infection events, however, the number of latently infected cells will also be reduced in the face of stronger immunity. In the model, latently infected cells continuously re-activate to give rise to productively infected cells. However, productive infection is kept at bay by the ongoing CTL memory response. Note that CTL inflation dynamics cannot be reproduced by this model alone.

\subsection{Adding NK cell responses to the model}

Here we add an innate immune response, denoted by $z_{\mathrm{i}}$. In particular, we consider the NK cell response. This is because NK cells play a critical role in the innate response against $\mathrm{CMV}$ infection, and initially control
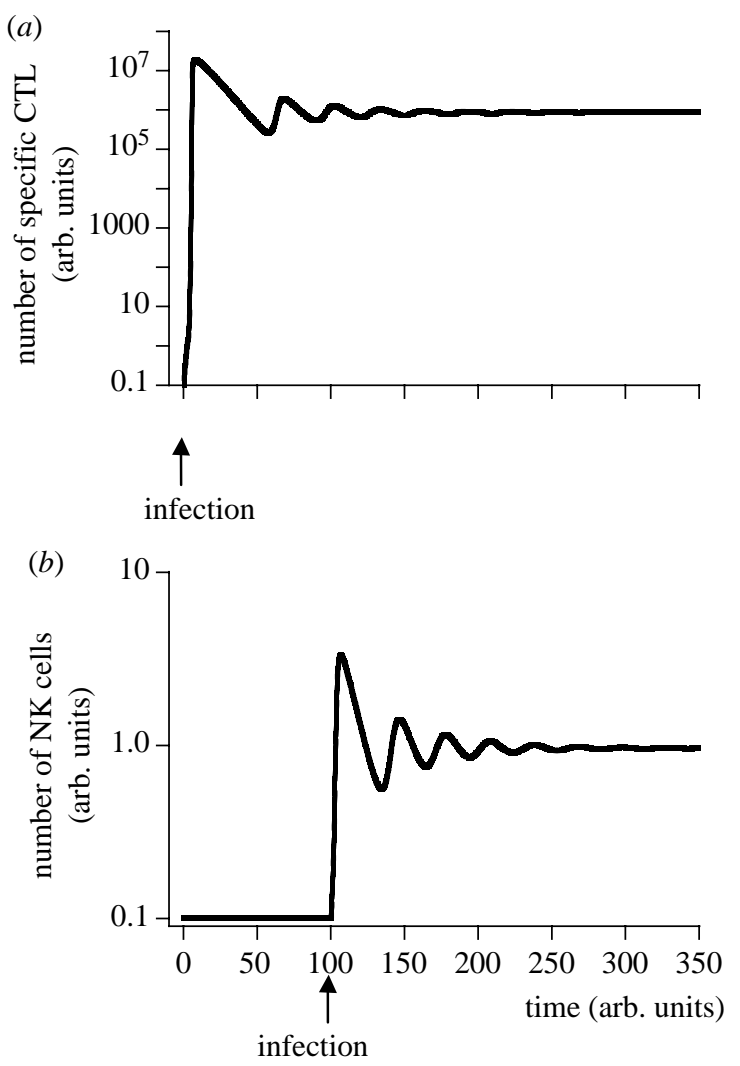

Figure 2. Immune response dynamics in CMV infection predicted by computer simulations. The timing of infection is indicated by an arrow. Two immune responses are considered: the CTL response and the NK cell response. In the simulation for the NK cell response, we have the infection start at a later time point in the simulation compared with the CTL response. This is because we would like to emphasize that a significant number of NK cell effectors exist independent from antigenic stimulation and are ready to fight the infection immediately as the virus enters the host. This is not true for CTL, which have to undergo clonal expansion and differentiation first. Further note that the units of the numbers of immune cells are arbitrary and should not be compared. (a) CTL response. Before the infection, specific CTL do not pre-exist as effectors. Instead a small number of naive CTL are present. Upon antigenic stimulation, the naive CTL become activated, expand and develop effector function. Subsequently, the CTL response declines until it settles around a stable level. This marks the chronic phase where an ongoing CTL response contains a persistent virus population. (b) NK cell response. Before infection, NK cells that can potentially attack the virus pre-exist. If this effector activity is too strong and reduces the basic reproductive ratio of the virus below one, the infection cannot become established in the host. In this simulation, this is not the case and a persistent infection is established. Consequently, the NK cell population expands in response to antigenic stimulation and settles around an equilibrium during chronic infection. The number of NK cells does not fall back to pre-infection levels because the persisting virus keeps boosting the cells. Parameters were chosen as follows: $\lambda=10, d=0.1, \beta=0.1$, $a_{0}=0.1, a_{1}=0.2, k=1, u=1, p_{\mathrm{a}}=0.000001, c_{\mathrm{a}}=15.5, p_{\mathrm{i}}=1$, $c_{\mathrm{i}}=12, b=0.1, \gamma=0.5, \phi=0.1, \eta=0.01, r=1, \delta=10, \xi=0.01$.

viral replication in the target organs. Data indicate that there are two important components to an NK cell response (Brown et al. 2001; Daniels et al. 2001; Lee et al. 2001). First, many of the NK cells which exist before the infection can kill virus-infected cells because 
they are recognized by a number of different receptors. Second, NK cells bearing specific receptors can expand in response to antigenic stimulation (Dokun et al. 2001). Therefore, we assume that a certain number of NK cells with the potential to kill CMV infected cells exist before infection, and in addition the NK cell population proliferates when antigen is encountered. We assume that antigen is recognized on infected cells but that latently infected cells are not recognized. The model which includes the NK cell response is described schematically in figure 1 and is written out in $\S 2$. NK cells are produced with a constant rate $\xi$ and die with a rate $b_{\mathrm{i}}$. Thus, in the absence of infection, the number of NK cells which can recognize and kill CMV infected cells is given by $\xi / b_{\mathrm{i}}$. In addition, the presence of the virus can lead to the proliferation of the NK cells with a rate $c_{\mathrm{i}}$. NK cells kill infected cells (those which express early or late-viral gene products) with a rate $p_{\mathrm{i}}$. Similarly to CTL, NK cells are known to also inhibit viral replication via the secretion of cytokines. Again, extensive numerical simulations indicate that our results do not depend on the particular mode of antiviral activity. Therefore, only killing was included in the model for simplicity. The main difference to the CTL model is that a reactive population of cells already exists before infection which is ready to fight the virus as soon as it enters the host.

In the absence of a CTL response, the typical dynamics are as follows. Initial virus growth is immediately countered by the NK cells. If the strength of the NK cells lies above a threshold, the virus infection cannot become established (the basic reproductive ratio of the virus is essentially less than one). If NK cell mediated killing is less strong, then the basic reproductive ratio of the virus is greater than one and a productive infection is established. Virus growth induces expansion of the NK cell population. The number of NK cells peaks, subsequently declines and settles at a steady state which describes a controlled but persistent infection (figure $2 b$ ). The stronger the NK cell response, the lower the number of infected cells and the amount of free virus.

\subsection{NK cell-CTL interactions}

We observe more complex dynamics if both the NK cell and the CTL response develop. Now, there is a degree of competition for antigenic stimulation between CTL and NK cells. This is because one branch of immunity can suppress virus load and compromise the other. However, this competition is asymmetric. The development of CTL effector activity depends on antigenic stimulation. The NK cell response can significantly suppress virus load and thereby compromise the development of CTL effector activity. CTL can also suppress virus load. However, the generation of NK cell effector activity is not compromised significantly by CTL. This is because a population of reactive NK cells exists even before the infection, and immediate effector activity does not rely on antigen-induced expansion of the NK cell population. Thus, in the following we will examine how the CTL dynamics depend on NK cell activity in the model.
We vary the strength of the NK cell response from high to low. The strength of the NK cell response is captured by the number of reactive NK cells which preexist before the infection (given by $\xi / b_{\mathrm{i}}$ ), their effector activity, $p_{\mathrm{i}}$, and their proliferation rate, $c_{\mathrm{i}}$. As mentioned above, if there are sufficient number of reactive NK cells which pre-exist before the start of the infection, and if they kill infected cells at a sufficiently high rate, then the basic reproductive ratio of the virus is less than one and an infection is not established. Consequently, a CTL response is not established either. Now assume that the NK cell response cannot prevent the establishment of infection. We observe an initial growth phase of the virus population, the extent of which depends on how effective the pre-existing NK cells are at removing infected cells. The NK cell population will also expand in response to antigenic stimulation. In addition, the population of CMVspecific CTL will become stimulated and expand. In the following, we examine how the dynamics of the CTL response depend on the strength of the NK cell response. We will concentrate on the rate of NK cell proliferation (or NK cell responsiveness, $c_{\mathrm{i}}$ ) as a measure of NK cell efficacy. We distinguish between three basic types of CTL dynamics.

(i) The NK cell responsiveness is relatively high and lies above a threshold (figure $3 a$ ). We observe an initial expansion phase of the CTL, followed by a decline. This decline will eventually result in the extinction of the CTL population, despite an ongoing persistent infection. The reason is that the NK cell response suppresses virus load to levels which are too low to maintain the CTL response. Whether the specific CTL will go extinct in practice depends on the lifespan of memory CTL. Experiments suggest that memory CTL can be maintained for long periods of time in the absence of antigenic stimulation. In this case, the CTL would not go extinct in a realistic period of time, but persist as a population of resting memory CTL which decline at a very slow rate (figure $3 a$ ).

(ii) The NK cell response is weaker, and antigen continues to re-stimulate the CTL in the long term (figure $3 b$ ). We again observe an initial expansion of the CTL population, followed by a contraction phase. Whereas, it's now the contraction phase is followed by a steady increase of the number of specific CTL during the chronic phase of the infection (figure $3 b$ ). This may correspond to the CTL inflation dynamics. The reason is as follows. The NK cell response is initially more effective than the CTL because it can achieve higher levels of lysis at the initial stage. Thus, NK cells play the dominant role in this acute phase and downregulate the virus population. This prevents the CTL from expanding fully. Consequently, they contract in the face of limited antigenic stimulation-i.e. effectively prematurely. The dynamics between the virus population and the NK cells oscillates to a steady state. Virus load at this steady state 

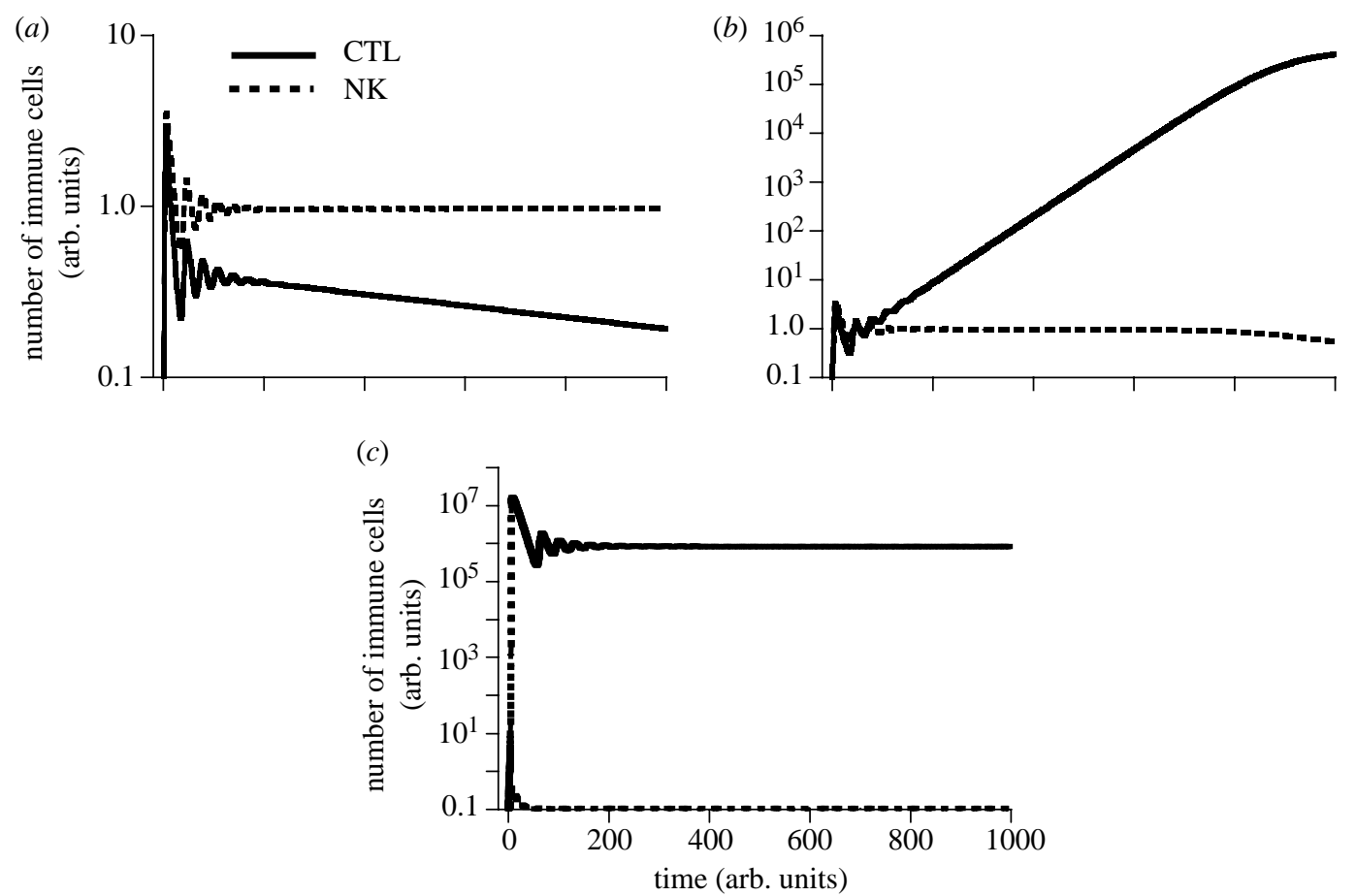

Figure 3. Competition dynamics between CTL and NK cells. The competition dynamics depend on the relative effectiveness of the CTL and NK cell responses at controlling the infection. (a) NK cells are the dominant immunological force and control the virus throughout the infection. The CTL expand and decline during acute infection and subsequently settle around a memory level at which they decline with a very slow rate. These may be resting memory cells. The reason is that during chronic infection, virus load is too low to stimulate the CTL in this case. (b) NK cells are the dominant controlling response during acute infection, but post-acute virus load is high enough to allow for CTL expansion during latent infection. The reason is as follows. NK cells which can potentially fight the virus already pre-exist as the virus enters the host. Upon antigenic stimulation, the NK cells also expand and the virus population is quickly driven to a relatively low steady state. The CTL response also reacts during acute infection. Because no effector cells pre-exist when the virus enters the host, the development of CTL-mediated activity is slower than the development of NK-mediated activity. Thus, acute virus load is controlled by the NK cells. Consequently, the CTL do not fully expand and decline again. However, because NK fail to reduce virus load to sufficiently low levels during chronic infection, antigen-driven CTL expansion starts to occur again. This continues until the CTL have reached a level at which the overall CTL-mediated activity has a stronger effect on the virus population than the NK cell mediated activity. At this point, the number of NK cells declines to a certain degree. This last stage of the dynamics might not be observed in vivo. These dynamics could correspond to CTL inflation. (c) In this simulation, the NK cell response is ineffective at controlling the virus population, even in the acute phase. Therefore, the CTL are immediately the dominant response, reduce the virus population and settle around a stable level at which they control the persistent infection. No CTL inflation is observed. This may reflect the situation where no significant amount of NK cell effectors are present in the host. Parameters were chosen as follows: $\lambda=10, d=0.1, \beta=0.1$, $a_{0}=0.1, a_{1}=0.2, k=1, u=1, p_{\mathrm{a}}=0.000001, c_{\mathrm{a}}=15.5, p_{\mathrm{i}}=1, b=0.1, \gamma=0.5, \phi=0.1, \eta=0.01, r=1, \delta=10, \xi=0.01$. For $(a) c_{\mathrm{i}}=14$, for $(b) c_{\mathrm{i}}=12$, and for $(c) c_{\mathrm{i}}=1$.

is high enough so that the CTL become stimulated and expand during the chronic phase. CTL expansion continues until the number of CTL is high enough such that CTLmediated killing becomes the dominant immune effector mechanism. Then the CTL settle around a steady state and control the virus population (figure $3 b$ ). At the same time, the NK cell response is expected to decline to a certain extent. These end stage dynamics might only occur after a time period too long to be observed in vivo. The inflation dynamics are determined by the following factors. The strength of NK cell mediated virus control can determine the degree of inflation. The weaker the NK cell response, the faster the rate of inflation. That is, the CTL population expands relatively slowly if the NK cell population is stronger, and faster if the NK cell response is weaker. The weaker the NK cell response, the higher the antigenic drive during chronic infection, and this allows for more pronounced CTL expansion (figure 4). Another factor which influences the amount of inflation can be the rate of CTL-mediated effector activity. CTL inflation is more pronounced if the rate of CTL-mediated activity is weaker. This is because with weaker CTL-mediated activity, a higher number of specific CD8 T cells are required to achieve CTL-mediated control of the virus population.

(iii) Finally, if the NK cell response is less effective at reducing acute virus load compared with the CTL response, we do not observe CTL inflation dynamics (figure $3 c$ ). In this case, CTL settle around a stable memory level after the acute phase of the infection. The difference to the first scenario described above is that now the memory cells are expected to be activated and not resting. The reason is that NK cells now do not play a significant role in limiting acute virus 


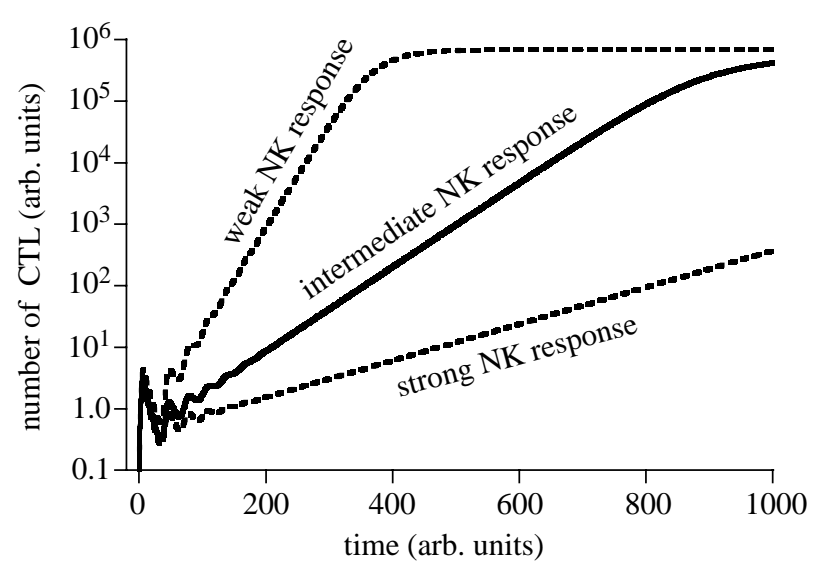

Figure 4. Degree of CTL inflation depends on the protectiveness of the NK cell response. We concentrate on the parameter region where CTL inflation is possible, i.e. the parameter region chosen for figure $3 b$, and vary the relative strength of the NK cell response within this region. The weaker and less protective the NK cell response, the stronger the degree of CTL inflation which is observed. The reasons are as follows. The weaker the NK cells, the less virus load is reduced after acute infection. The less virus load is reduced, the higher the amount of antigenic stimulation for the CTL during chronic infection, and the faster the rate of $\mathrm{CTL}$ expansion. As mentioned in figure 3, if the protectiveness of NK cells falls below a threshold and is too low, such that CTL are more effective at reducing virus load already during acute infection, then CTL inflation does not occur and these considerations do not hold. Parameters were chosen as follows: $\lambda=10, d=0.1, \beta=0.1, a_{0}=0.1, a_{1}=0.2, k=1, u=1$, $p_{\mathrm{a}}=0.000001, c_{\mathrm{a}}=15.5, p_{\mathrm{i}}=1, \quad b=0.1, \gamma=0.5, \phi=0.1, \eta=$ $0.01, r=1, \delta=10, \xi=0.01$. For weak strong NK response $c_{\mathrm{i}}=$ 13 , for intermediate NK response $c_{\mathrm{i}}=12$, and for weak NK response $c_{\mathrm{i}}=10$.

growth. Consequently, the CTL are immediately the dominant immune response which drive the dynamics during acute infection and suppress virus load.

We would like to point out that in the graphs above, the effect of competition is only observed after CTL responses have peaked. This is because we assume that CTL respond by a proliferation program that is independent from antigenic stimulation (Kaech \& Ahmed 2001; van Stipdonk et al. 2001). Therefore, the peak of the CTL response will not reflect effects of competition. However, when CTL become re-stimulated during the longer term dynamics, competition clearly influences the time course of the CTL response.

In summary, the dynamical interplay between NK cells, CTL and virus population can lead to the phenomenon of CTL inflation. If NK cells are more efficient than CTL at reducing virus load during acute infection, but fail to keep virus load at sufficiently low levels during chronic infection, then we expect that CTL first expand to a limited peak, contract and then inflate. A major factor which determines the extent of CTL inflation is the strength of the NK cell mediated control of chronic virus load. If the NK cells response is strong, it keeps the virus at relatively low levels during the chronic phase of the infection. This provides only a limited antigenic stimulus for the expansion of CTL, and the extent of CTL inflation is low. A weaker NK cell response allows higher virus loads during the chronic phase of the infection. This allows for a higher antigenic stimulus for the CTL. Consequently, CTL inflation is more pronounced. In addition, the weaker the rate of CTL-mediated effector activity the larger the degree of inflation, because more specific CD8 T cells are required to control the virus population; this latter relationship may differ significantly even between responses to epitopes which escape immunoevasins and are presented during the phase of clinical latency. The prediction that the extent of NK cell mediated protection can shape the dynamics of CTL memory inflation is supported by experimental data from MCMV infected mice. This is shown $\$ 3.5$.

\subsection{CTL inflation and NK cell responses: experimental data}

To address whether NK cells have an effect on the $\mathrm{CD} 8+\mathrm{T}$ cell inflation, C57BL/6 mice were infected with a recombinant MCMV expressing a peptide derived from the lymphocytic choriomeningitis virus (LCMV) glycoprotein (Karrer et al. 2004). This peptide (gp 31-42) was fused to the C-terminus of the nonessential ie2 gene of MCMV and is therefore expressed during latency under the control of the immediate early promotor (Grzimek et al. 2001). This induced a subdominant but inflationary gp34-specific $\mathrm{CD}^{+} \mathrm{T}$ cell response as monitored by tetramer. C57BL/6 were either depleted of NK1.1 cells or left untreated before MCMV-GP infection and the expansion of gp34specific $\mathrm{CD}^{+} \mathrm{T}$ cells over time was measured in the blood (figure $5 a$ ). As predicted by the model we observed a bigger CD8 $+\mathrm{T}$ cell expansion when mice were depleted of NK cells during the acute phase of infection. Note that although NK cells were depleted in this experiment, this depletion occurred before infection and was transient. Therefore, this scenario does not correspond to the complete absence of NK cells in which case we would not expect inflation dynamics to occur. While this experiment manipulated the number of NK cells that are able to fight the virus upon infection, we varied the 'responsiveness' of the NK cells in our simulations (figures 3 and 4). Extensive simulations, however, indicate that the effect is the same, as in both cases the ability of the NK cells, to initially take control of the infection, is changed.

Resistance to MCMV is associated with the presence of Ly $49 \mathrm{H}$, an activating NK cell receptor in some mouse strains (Daniels et al. 2001; Lee et al. 2001). Ly49H binding to m157, a viral protein, activates $\mathrm{Ly} 49 \mathrm{H}$ expressing NK cells and leads to increased resistance to MCMV (Arase et al. 2002). In C57BL/6, approximately $50 \%$ of NK cells express Ly $49 \mathrm{H}$ rending those mice more resistant to MCMV. On the other hand, BALB/B mice, which express the same MHC haplotype as $\mathrm{C} 57 \mathrm{BL} / 6$ (H-2b), lack Ly49H expressing NK cells and are much more susceptible MCMV infection. We therefore infected C57BL/6 and BALB/B mice with $10^{6} \mathrm{pfu}$ of MCMV-GP and followed the expansion of gp34-specific $\mathrm{CD}^{+} \mathrm{T}$ cells over time. We observed a much more pronounced expansion of gp34 + specific CD8 $+\mathrm{T}$ cells 

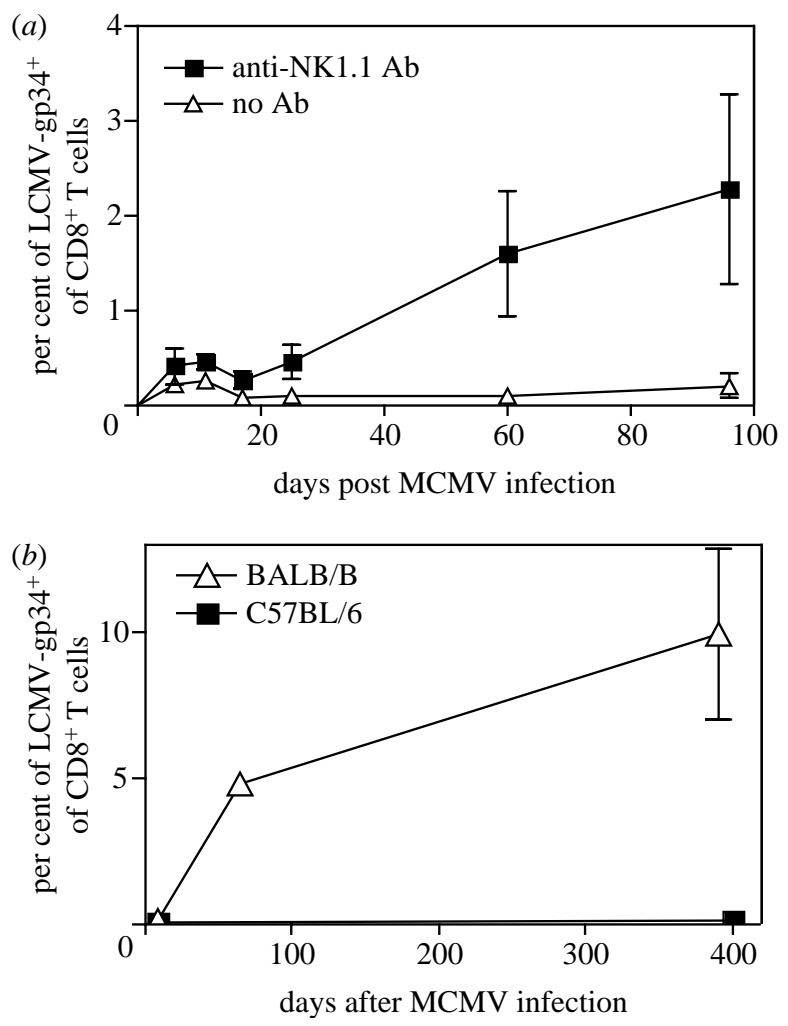

Figure 5. Increased CD8 $+\mathrm{T}$ cell inflation in the absence of NK cells during acute MCMV infection. (a) C57BL/6 naive mice or C57BL/6 mice treated with PK136 mAb to deplete NK1.1 cells were infected with $10^{6}$ pfu of MCMV-GP intravenously. (b) C57BL/6 and BALB/B mice were infected with $10^{6}$ pfu MCMV-GP intravenously. At the indicated time points, the mice were bled and peripheral blood cells were stained with anti-CD8 and Kb-gp34 tetramer. The percentage of CD8 + T cells staining with the Kb-gp34 tetramer is plotted.

in BALB/B mice over time as compared with $\mathrm{C} 57 \mathrm{BL} / 6$ animals (figure $5 b$ ). This is again consistent with model predictions.

Finally, in a parallel study we compared the dynamics of the immune response with MCMV in two strains of BALB/c mice which are congenic for the Ly49H receptor (Sierro et al. in preparation). In this experiment the mice are identical, but for the presence of the protective NK population, and this leads to a much-diminished inflationary profile in the long term.

While these experiments support the notions which were derived from the mathematical model, these are preliminary experimental studies. The correlation between the occurrence of CTL inflation and the status of both Ly49H + and Ly49H- NK cell responses needs to be investigated experimentally in a more rigorous fashion in order to draw stronger conclusions. However, this would go beyond the scope of the current paper which is largely theoretical in nature. However, the results described here provide a solid framework for further experimental investigation.

\section{CONCLUSION}

We have used a mathematical model which describes the dynamical interactions between CMV and the immune system in order to propose an explanation for the phenomenon of CTL memory inflation. The model argues that the competitive interactions between NK cells and CTL can give rise to inflation dynamics. This requires that the NK cells are more efficient at reducing virus load during the acute phase of the infection, but maintain virus loads during chronic infection at levels which are sufficient for CTL to become stimulated and expand. Experimental data which examine the correlation between NK-mediated protection and CTL inflation support the theoretical predictions.

The fact that experimental data are in agreement with theoretical predictions, however, does not prove our explanation to be correct. At this stage, it remains a hypothesis that provides stimulation for further experimental work. Our modelling suggests that the simplest mechanism put forward so far, that is, long-term re-stimulation of CTL during latent infection brought about by periodic reactivation of latent cells, cannot produce the inflation dynamics that are observed in experiments. Additional mechanisms must be at work, and we have suggested one that is consistent with the data collected so far. If further hypotheses are brought forward in the context of alternative models, experiments can be used to distinguish between them. One variant of our hypothesis could be that it is not the competition between CTL and NK cells in particular that accounts for the inflation dynamics, but the competition between CTL and other branches of immunity in general. According to numerical simulations, even the competition between CTL clones directed against different epitopes (De Boer \& Perelson 1994; Nowak et al. 1995) can result in some CTL clones inflating over time while others remain steady. This can occur if one CTL clone is more efficient during the initial stages of the infection, while the other CTL clone is more efficient during the latent phase of the infection. However, the competition between CTL and NK cells seems to be the most likely candidate mechanism because NK effectors are present in the absence of antigen, even before the infection, and CTL effectors are not. Thus, NK cells are likely to be more efficient in the very early stages of the infection, setting the stage for the inflation dynamics. In addition to these theoretical arguments, the experimental data discussed here strongly point to an involvement of NK cells in CTL inflation.

It will be interesting to see whether inflation dynamics are observed in the context of any other virus infections, and whether a similar mechanism can be responsible. Recent data from acute parvovirus B19 infection in man demonstrate that memory inflation can occur during the first 12-18 months of infection, at a timepoint where virus is no longer readily detectable in blood (Isa et al. 2005; Norbeck et al. 2005). These $\mathrm{CD} 8+\mathrm{T}$ cell populations show signs of activation and maturation similar to, if not exceeding, those in CMV, suggesting re-encounter with antigen over this period, although little data are available on NK cell responses over this period.

While the model has only considered a single CTL response, multiple CTL clones directed against different epitopes are observed in vivo. Depending on the CTL responsiveness and the rate of CTL-mediated 
effector activity, some CTL responses may inflate while others do not. A more complex model that includes multiple CTL clones directed against different epitopes can reproduce this behaviour (not shown). For example, the amount of fully replicative virus maintained by the NK cell response may be too little to provide antigenic stimulation for some CTL clones during chronic infection, while it will be sufficient for others. Consequently some clones might remain as resting memory cells at a stable level, while others inflate. This has been observed in experimental data from MCMV infected mice (Karrer et al. 2003). Recent data from Munks et al. and Sierro et al. do point to the existence of quite distinct pathways of memory in latent CMV. A set of immune responses do not appear to undergo restimulation over this period and appear to be 'blind' to the presence of virus. A fuller understanding of how, where and when immunoevasins encoded by the virus block presentation of specific gene products (but not others) is required - however, it is clear that the dynamics discussed in this paper will apply to only a subset of responses, albeit those which dominate dramatically in memory pools.

The model behaviour does not depend strictly on latent virus. During the acute response the NK cell response is dominant and subsequently the persisting virus stimulates the CTL. The weaker the NK response, the more virus remains and the stronger the stimulation and inflation. Inflation would depend strictly on latently infected cells only if the virus was eradicated by NK cells alone, in the absence of latency. In this model both NK cells and CTL are responsible for the control of the virus during the latent phase of infection.

The experimental data presented here are in accordance with the model: we followed the inflation of gp34-specific CD8 + T cells during the latent phase of infection in different NK cell environments. Depletion of NK cells during the acute phase of infection, although only transient, does influence the subsequent $\mathrm{CD} 8^{+} \mathrm{T}$ cell inflation during the early latent phase of infection. Infection of BALB/B mice which have a much weaker MCMV generated NK cell response compared to C57BL/6 also support the idea that the strength of the NK response influences the inflation of $\mathrm{CD} 8^{+} \mathrm{T}$ cells during the latent phase. Although in this case, we cannot exclude the possibility that other factors due to the different genetic backgrounds of the mice may also play a role. A further study in our lab, using a more controlled comparison between congenic mouse strains has also highlighted critical differences in the late evolution of memory due to the presence of highly efficient NK responses. In this case the presence of more efficient NK cells expressing the Ly49H receptor leads to diminution of CD8+ T cell inflation (Sierro et al. in preparation), consistent with the idea that the two populations are effectively in competition.

The model is also relevant to human CMV infection. Although this is well controlled in normal individuals and latency is maintained lifelong, this is a significant clinical problem in the immunosuppressed. Interestingly, recent data do suggest that very large expansions of CMV specific CTL exist in normal individuals and that these gradually expand with age (Khan et al. 2002,
2004; Komatsu et al. 2003). It is suggested also that such populations may be associated with reduced expansions of $\mathrm{T}$ cell responses against other viruses, as well as vaccines. Such 'immunoparesis' could be associated with the excess death rate seen in seropositive elderly individuals. Importantly, a wide variation in immune responses against a single epitope is seen among individuals sharing a common HLA molecule. We speculate that variation in host-innate responses (such as polymorphisms in inherited NK receptors) could play a significant role in this inter-host variability.

This study was funded in part by NIH grant R01 AI058153$01 \mathrm{~A} 2$.

\section{REFERENCES}

Altman, J. D., Moss, P. A., Goulder, P. J., Barouch, D. H., McHeyzer-Williams, M. G., Bell, J. I., McMichael, A. J. \& Davis, M. M. 1996 Phenotypic analysis of antigen-specific T lymphocytes. Science 274, 94-96. (doi:10.1126/science. 274.5284.94)

Anderson, R. M. \& May, R. M. 1991 Infectious diseases of humans. Oxford, UK: Oxford University Press.

Antia, R., Bergstrom, C. T., Pilyugin, S. S., Kaech, S. M. \& Ahmed, R. 2003 Models of CD8 + responses: 1. What is the antigen-independent proliferation program. J. Theor. Biol. 221, 585-598. (doi:10.1006/jtbi.2003.3208)

Arase, H., Mocarski, E. S., Campbell, A. E., Hill, A. B. \& Lanier, L. L. 2002 Direct recognition of cytomegalovirus by activating and inhibitory NK cell receptors. Science 296, 1323-1326. (doi:10.1126/science.1070884)

Brown, M. G. et al. 2001 Vital involvement of a natural killer cell activation receptor in resistance to viral infection. Science 292, 934-937. (doi:10.1126/science.1060042)

Bunde, T. et al. 2005 Protection from cytomegalovirus after transplantation is correlated with immediate early 1-specific CD8 T cells. J. Exp. Med. 201, 1031-1036. (doi:10.1084/jem.20042384)

Daniels, K. A., Devora, G., Lai, W. C., O'Donnell, C. L., Bennett, M. \& Welsh, R. M. 2001 Murine cytomegalovirus is regulated by a discrete subset of natural killer cells reactive with monoclonal antibody to Ly49H. J. Exp. Med. 194, 29-44. (doi:10.1084/jem.194.1.29)

De Boer, R. J. \& Perelson, A. S. 1994 T cell repertoires and competitive exclusion. J. Theor. Biol. 169, 375-390. (doi:10.1006/jtbi.1994.1160)

De Boer, R. J. \& Perelson, A. S. 1998 Target cell limited and immune control models of HIV infection: a comparison. J. Theor. Biol. 190, 201-214. (doi:10.1006/jtbi.1997.0548)

Dokun, A. O., Kim, S., Smith, H. R., Kang, H. S., Chu, D. T. \& Yokoyama, W. M. 2001 Specific and nonspecific NK cell activation during virus infection. Nat. Immunol. 2, 951-956. (doi:10.1038/ni714)

Grzimek, N. K., Dreis, D., Schmalz, S. \& Reddehase, M. J. 2001 Random, asynchronous, and asymmetric transcriptional activity of enhancer-flanking major immediate-early genes ie $1 / 3$ and ie 2 during murine cytomegalovirus latency in the lungs. J. Virol. 75, 2692-2705. (doi:10.1128/JVI.75. 6.2692-2705.2001)

Guidotti, L. G., Borrow, P., Brown, A., McClary, H., Koch, R. \& Chisari, F. V. 1999 Noncytopathic clearance of lymphocytic choriomeningitis virus from the hepatocyte. J. Exp. Med. 189, 1555-1564. (doi:10.1084/jem.189.10. 1555) 
Holtappels, R., Thomas, D., Podlech, J. \& Reddehase, M. J. 2002 Two antigenic peptides from genes m123 and m164 of murine cytomegalovirus quantitatively dominate CD8 T-cell memory in the H-2d haplotype. J. Virol. 76, 151-164. (doi:10.1128/JVI.76.1.151-164.2002)

Hudrisier, D., Oldstone, M. B. \& Gairin, J. E. 1997 The signal sequence of lymphocytic choriomeningitis virus contains an immunodominant cytotoxic $\mathrm{T}$ cell epitope that is restricted by both $\mathrm{H}-2 \mathrm{D}(\mathrm{b})$ and $\mathrm{H}-2 \mathrm{~K}(\mathrm{~b})$ molecules. Virology 234, 62-73. (doi:10.1006/viro.1997.8627)

Isa, A., Kasprowicz, V., Norbeck, O., Loughry, A., Jeffery, K., Broliden, K., Klenerman, P., Tolfvenstam, T. \& Bowness, P. 2005 Prolonged activation of virus-specific CD8+T cells after acute B19 infection. PLoS Med. 2, e343. (doi:10. 1371/journal.pmed.0020343)

Kaech, S. M. \& Ahmed, R. 2001 Memory CD8+T cell differentiation: initial antigen encounter triggers a developmental program in naive cells. Nat. Immunol. 2, 415-422.

Kaech, S. M., Wherry, E. J. \& Ahmed, R. 2002 Effector and memory T-cell differentiation: implications for vaccine development. Nat. Rev. Immunol. 2, 251-262. (doi:10. 1038/nri778)

Karrer, U., Sierro, S., Wagner, M., Oxenius, A., Hengel, H., Koszinowski, U. H., Phillips, R. E. \& Klenerman, P. 2003 Memory inflation: continuous accumulation of antiviral CD8 + T cells over time. J. Immunol. 170, 2022-2029.

Karrer, U. et al. 2004 Expansion of protective CD8+T-cell responses driven by recombinant cytomegaloviruses. J. Virol. 78, 2255-2264. (doi:10.1128/JVI.78.5.2255-2264. 2004)

Khan, N., Shariff, N., Cobbold, M., Bruton, R., Ainsworth, J. A., Sinclair, A. J., Nayak, L. \& Moss, P. A. 2002 Cytomegalovirus seropositivity drives the CD8 T cell repertoire toward greater clonality in healthy elderly individuals. J. Immunol. 169, 1984-1992.

Khan, N., Hislop, A., Gudgeon, N., Cobbold, M., Khanna, R., Nayak, L., Rickinson, A. B. \& Moss, P. A. 2004 Herpesvirus-specific CD8 T cell immunity in old age: cytomegalovirus impairs the response to a coresident EBV infection. J. Immunol. 173, 7481-7489.

Komatsu, H., Sierro, S., A, V. C. \& Klenerman, P. 2003 Population analysis of antiviral $\mathrm{T}$ cell responses using MHC class I-peptide tetramers. Clin. Exp. Immunol. 134, 9-12. (doi:10.1046/j.1365-2249.2003.02266.x)

Kurz, S. K., Rapp, M., Steffens, H. P., Grzimek, N. K., Schmalz, S. \& Reddehase, M. J. 1999 Focal transcriptional activity of murine cytomegalovirus during latency in the lungs. J. Virol. 73, 482-494.

Lee, S. H., Girard, S., Macina, D., Busa, M., Zafer, A., Belouchi, A., Gros, P. \& Vidal, S. M. 2001 Susceptibility to mouse cytomegalovirus is associated with deletion of an activating natural killer cell receptor of the C-type lectin superfamily. Nat. Genet. 28, 42-45. (doi:10.1038/88247)
Mercado, R., Vijh, S., Allen, S. E., Kerksiek, K., Pilip, I. M. \& Pamer, E. G. 2000 Early programming of T cell populations responding to bacterial infection. J. Immunol. 165, 6833-6839.

Munks, M. W., Cho, K. S., Pinto, A. K., Sierro, S., Klenerman, P. \& Hill, A. B. 2006 Four distinct patterns of memory $\mathrm{CD} 8 \mathrm{~T}$ cell responses to chronic murine cytomegalovirus infection. J. Immunol. 177, 450-458.

Norbeck, O., Isa, A., Pohlmann, C., Broliden, K., Kasprowicz, V., Bowness, P., Klenerman, P. \& Tolfvenstam, T. 2005 Sustained CD8+T-cell responses induced after acute parvovirus B19 infection in humans. J. Virol. 79, 12 117-12 121. (doi:10.1128/JVI.79.18.1211712121.2005)

Nowak, M. A. \& Bangham, C. R. 1996 Population dynamics of immune responses to persistent viruses. Science 272, 74-79. (doi:10.1126/science.272.5258.74)

Nowak, M. A. et al. 1995 Antigenic oscillations and shifting immunodominance in Hiv-1 infections. Nature $\mathbf{3 7 5}$, 606-611. (doi:10.1038/375606a0)

Perelson, A. S. 2002 Modelling viral and immune system dynamics. Nat. Rev. Immunol. 2, 28-36. (doi:10.1038/ nri700)

Reddehase, M. J. 2002 Antigens and immunoevasins: opponents in cytomegalovirus immune surveillance. Nat. Rev. Immunol. 2, 831-844. (doi:10.1038/nri932)

Seaman, W. E., Sleisenger, M., Eriksson, E. \& Koo, G. C. 1987 Depletion of natural killer cells in mice by monoclonal antibody to NK-1.1. Reduction in host defense against malignancy without loss of cellular or humoral immunity. J. Immunol. 138, 4539-4544.

Sierro, S., Rothkopf, R. \& Klenerman, P. 2005 Evolution of diverse antiviral $\mathrm{CD} 8+\mathrm{T}$ cell populations after murine cytomegalovirus infection. Eur. J. Immunol. 35, 1113-1123. (doi:10.1002/eji.200425534)

Sierro, S. et al. In preparation.

van Stipdonk, M. J., Lemmens, E. E. \& Schoenberger, S. P. 2001 Naive CTLs require a single brief period of antigenic stimulation for clonal expansion and differentiation. Nat. Immunol. 2, 423-429.

Wherry, E. J. \& Ahmed, R. 2004 Memory CD8 T-cell differentiation during viral infection. J. Virol. 78, 5535-5545. (doi:10.1128/JVI.78.11.5535-5545.2004)

Wodarz, D. \& Nowak, M. A. 2000 Immune responses and viral phenotype: do replication rate and cytopathogenicity influence virus load? J. Theor. Med. 2, 113-127.

Wodarz, D. \& Thomsen, A. R. 2005 Effect of the CTL proliferation program on virus dynamics. Int. Immunol. 17, 1269-1276. (doi:10.1093/intimm/dxh303)

Wodarz, D., Christensen, J. P. \& Thomsen, A. R. 2002 The importance of lytic and nonlytic immune responses in viral infections. Trends Immunol. 23, 194-200. (doi:10.1016/ S1471-4906(02)02189-0) 